to granulate with a view to covering it ultimately by skin transplantation.

Three weeks later we found that a little sloughing had taken place on both sides, where the tension was greatest; but the penis was quite released from its abnormal surroundings, and there seemed every prospect of the granulations being covered by skin-grafting in a manner which would obviate any further obstruction to the movements of the lower limbs.

\section{EXCISION OF THE HEAD OF THE FEMUR.}

On Wednesday last Mr. Gay excised the head of the femur in a child five years of age, who had disease of the hip of twelve months' standing, and was admitted in a state of exhaustion, with numerous pus-discharging openings in the thigh and groin. The capsule had been opened by the disease, but dislocation had not taken place. A small piece of necrosed bone was liberated from the upper part of the acetabulum at the same time.

\section{MR. ADAMS'S CASE OF OSTEOTOMY.}

The general progress of this patient's case is satisfactory. With the exception of a rise of temperature on the day following the operation, there has been no constitutional disturbance. But only very limited movement of the femur independent of the pelvis has as yet been obtained, although the weight by which extension is being made has been gradually increased from three to eight pounds. This rigidity appears to be due, to a considerable extent, to insufficieney of skin on the anterior aspect-a condition which is scarcely to be wondered at when it is considered that, for ten years before the operation was undertaken, the femur had been maintained at such an angle that the trochanter major was on a level with the anterior inferior spine of the ilium. On the occasion of our last visit to this patient (the eighteenth day), there was no tenseness of integument at the point where the incision had been made, nor thickening in its track, and not a drop of pus could be squeezed from the minute aperture in the skin.

Mr. Adams proposes to attempt forcible extension under chloroform on Wednesday next.

\section{PROVINCIAL HOSPITAL REPORTS.}

\section{ST. MARY'S HOSPITAL, MANCHESTER. SEVEN GASES OF OVARIOTOMY. \\ Communicated by Mr. Hewry RUnconr, House-Surgeon. \\ (Under the care of Dr. LLoyd Rober'ts.)}

CASE 1. Removal of the right ovary, with a fibro-cystic tumour of the uterus weighing twenty-three pounds; recovery.-Margaret M- a - aged thirty-two, single, noticed the enlargement twenty-seven months before admission. It was not more marked on one side than the other. She had never been tapped. The abdomen measured forty-three inches in girth over the umbilicus, and from the ensiform cartilage to the pubes twenty-three inches. Fluctuation could be felt over the whole of the tumour. The uterus was movable, slightly hypertrophied, and was raised with the tumour when the latter was elevated by external manipulation.

Ovariotomy was performed under the influence of bichloride of methylene, administered by Dr. Junker's apparatus. An incision, three inches in length, was made down to the tumour, considerable hæmorrhage arising from the divided vessels of the abdominal wall, which was arrested by torsion. Some firm adhesions between the front of the tumour and abdominal parietes were separated, and a large syphon trocar was plunged twice into the tumour, but no fluid passed through it. The primary incision was enlarged to the extent of one inch above the umbilicus, and the tumour was brought through the abdominal wound. The pedicle, which was three inches broad and very short, was secured by a circular clamp. Some blood-clots were removed from the abdominal cavity, and the wound was brought together by five deep sutures of silk, and dressed with carbolised lotion and plaster, without the use of any abdominal bandage. With the exception of some dysuria on the sixth and seventh days, the patient had not a single unfavourable symptom. The clamp was removed on the sixteenth day, and a smaller one which was substituted was finally removed on the twentieth day. She left the hospital on the forty-sixth day.

The tumour when examined was found to consist of the right ovary in an early stage of cystic disease, with a fibrocystic tumour of the uterus. It was pedunculated. The diseased ovary and Fallopian tube, except its fimbriated extremity, were firmly united to the tumour.

CASE 2. Ovariotomy; unilocular ovarian cyst of the left side; ligature cut short; pedicle returned.-Elizabeth $\mathrm{P}-$ aged forty-nine, was a widow, and had had one living child and one miscarriage. The catamenia had ceased four years previously. A year after that event she noticed a swelling in the left side of the abdomen. On admission the abdomen was found to be large and prominent, yielding general fluctuation over the front and sides of the tumour and resonance over both flanks. The girth at the umbilicus was thirty-eight inches; from the ensiform cartilage to the pubes measured eighteen inches; the uterus was two inches and three-quarters lower in the pelvis than normal. Fluctuation was felt in Douglas's space on the left side. The patient had not been tapped.

The tumour was exposed and tapped with a large syphon trocar. The fluid withdrawn presented a dark grumous appearance. The tumour was then drawn through the wound, and the pedicle, a broad and flesby one an inch and a half in length, was secured by a ligature which was cut short, and, the tumour having been separated, the stump was dropped in to the abdomen. No fluid entered the peritoneal cavity. The abdominal wound was brought together by silk sutures, and dressed as in the above case. The incision was three inches in length.

With the exception of flatulence which troubled this patient for a few days, she presented no unfavourable symptom. The wound had healed by the seventh day, and on the eleventh she was convalescent. The tumour was unilocular, and with its contents weighed $19 \frac{2}{4} \mathrm{lb}$.

CASI 3. Multilocular ovarian tumour of the left side; ovariotomy with use of clamp; recovery. - Annie $\mathrm{B}-$, aged twenty-four, was married, but had had no children. She first menstruated at twelve years of age, and had been regular ever since. The swelling commenced five years before admission after a fall. The abdomen measured, over the umbilicus, forty inches; from the sternum to the pubes, twenty-one inches. The globular fluctuating tumour inclined to the left side. Fluctuation could be felt in both flanks. The uterus was normal in size, with a small cervix. The tumour compressed the bladder and caused frequent desire to urinate. She had never been tapped.

The operation was performed under the influence of bichloride of methylene. One large cyst was tapped in front, and a smaller cyst was then emptied. The two emptied cysts, and a number of smaller ones unopened, were drawn through the wound, and the pedicle, two and a half inches in length, was secured by a clamp. The abdominal incision was secured by silk sutures. The incision in this case was two inches in length. On the fourth day after the operation the patient had an urgent attack of dys. pncea with bronchial râles, which was subdued by a linctus, sinapisms, and brandy-and-water. The wound had healed by the eighteenth day, when the clamp was also removed.

CASE 4. Multilocular ovarian tumour of the left side, weighing twenty-seven pounds and a half; recovery. - Martha $\mathrm{H} \longrightarrow$, aged twenty-eight, was $\lambda$ married woman. The abdomen was much distended, irregular in shape, and bosse. lated. The fluctuation was localised; the swelling on the left side was solid; the linea alba was drawn towards the left side. The cyst on the right side reached to the ensiform cartilage, was conical in shape, and fluctuated distinctly. The girth at the umbilicus was forty-one inches and $a$ half; from the sternum to the pubes measured eighteen inches and a half. The uterus was slightly prolapsed, and the uterine sound passed to a distance of two inches and a half. Owing to the extent to which the breathing had been embarrassed by the tumour, the patient had not been able to lie in bed for three weeks. She had not been tapped.

The operation was performed under the influence of bichloride of methylene. After the abdomen had been opened in the usual manner, the bleeding which occurred from the vessels in the cut surfaces had been arrested, and 
the peritoneum divided, the right cyst came into view and was tapped. The fluid was dark-coloured and grumous. A second cyst was then tapped, and about three pints of a similar fluid were drawn off. Traction on the collapsed cysts having brought them outside the abdomen, a third came into view. This was drawn through the wound without being punctured, as well as a number of other smaller cysts. The pedicle, three inches long and an inch broad, connected with the left ovary, was secured by a clamp, and the tumour was then separated. The wound was brought together and dressed as in the previous cases. The sutures were all removed by the seventh day, the clamp came off on the eleventh day, and her recovery was uninterrupted from this time.

CASE 5. Removal of a multilocular ovarian tumour weighing forty-six pounds; firm adhesions; use of clamp; recovery.Belinda $\mathrm{H}-$, aged thirty-three, had had four children. The swelling commenced two years and eight months before admission. Eighteen pints of fluid had been removed by tapping three months previously. The abdominal walls were very tense, and fluctuation was very distinct. In the left iliac region was a solid substance which was very painful on pressure. There was considerable cedema of the abdominal parietes, both lower extremities, and the lower part of the back. No albumen was found in the urine. The girth at the umbilicus was forty-eight inches and threequarters, and, from the sternum to the pubes, measured twenty-three inches. The uterus was lower in the pelvis than normal, and the cervix was directed towards the lef side. The uterine sound passed to a distance of four inches and a half. The uterus was mobile.

Ovariotomy was performed under the influence of bichloride of methylene. The tumour was exposed by an incision three inches in length. There were firm adhesions between the tumour and the abdominal wall, which were with difficulty broken down. A cyst was then tapped and the fluid removed, and another cyst was emptied. The hand was then passed into the abdomen, and some firm adhesions which existed in the iliac and lumbar regions were broken down. Other adhesions above and behind were next separated. The incision was then lengthened upwards by one inch, and the tumour having been brought ontside, some omental adhesions were detached, and the pedicle (about three inches in length) connected with the left ovary was secured with a clamp and divided. The cyst-walls were so friable that in bringing out the tumour one of the unemptied cysts adherent to the abdominal wall gave way, and the contents flowed into the abdomen. A great deal of time was spent in clearing out the cystic fluid and blood from that cavity. The wound was brought together by five silk sutures.

This patient had not a single unfavourable symptom. The wound, except where the pedicle issued, was healed on the fifth day; the whole of the sutures and the clamp were removed by the twelfth day; and the patient made a rapid recovery.

Case 6. Solid ovarian tumour; ascites; ovariotomy; fatal result.-Susan R—, twenty.four, unmarried, first noticed the swelling two years before admission. She had been tapped about twelve months previously, and again in three months. The abdomen was tense and fluctuating, especially in the flanks, and was resonant in front to the right of the umbilicus. A distinct hard tumour could be felt superficially to occupy nearly a central position with the umbilicus for its centre, and to extend above, below, and slightly to the left of it. The girth of the abdomen was forty-two inches.

The tumour was firmly adherent to the diaphragm, omentum, and abdominal wall; a considerable time was required to separate the adhesions, and the pedicle, which was four inches long, narrow, and attached to the left ovary, was secured by a clamp, and the tumour separated. The ascitic fluid was then removed from the abdomen, and the wound closed with silk, as in the previous cases. 4 P.Mr. the patient had somewhat rallied after the operation. Her pulse was 120; she complained of great pain in the abdomen, which was relieved by a morphia suppository. She passed a very restless night, and on the following morning her pulse was 125 ; she dozed occasionally; still complained of severe pain in the abdomen, and was ordered iced brandy and iced milk. At 9 P.M. her pulse was 130 ; the abdominal pain had increased; she was very restless, and complained of flatulence. At 9 A.M. on the third day her pulse was 130 ; vomiting began during the morning and she sank exhausted at 5 P.Mr.

At the post-mortem examination the peritoneum, omentum, and intestines were much injected. About three quarts of bloody fluid in the abdominal cavity; right ovary healthy; uterus much atrophied; remains of adhesions seen in the omentum, diaphragm, and abdominal wall. The tumour weighed $41 \mathrm{~b} .2 \mathrm{oz}$. The ascitic fluid weighed $20 \frac{1}{2} \mathrm{lb}$.

\section{(Under the care of Mr. Walter Whitehead.)}

CASE 7. Removal of multilocular ovarian tumour weighing twenty-five pounds; recovery.-M. C-, aged fifty-nine, had been married thirty-seven years, and had borne seven living children and had two miscarriages. She first noticed an increase in the size of her abdomen about ten month 3 before admission, but could not remember that it was greater on one side than the other. She also observed, about the same time, repeated blood-stains on her linen, as though she had been menstruating, although that function had ceased sixteen years previously. The circumference round the umbilicus was forty-one inches.

Ovariotomy was performed under the influence of the bichloride of methylene. The tumour was removed by a small incision; uniform and extensive adhesions to the parietes were broken down, several cysts of variable size were emptied and drawn out, the remaining mass of semisolid matter and aggregation of small cysts were also drawn through the abdominal wound. The pedicle was secured by a clamp, and the wound closed by silk sutures. The tumour and contents weighed twenty-five pounds. The patient made a rapid and uninterrupted recovery.

\section{算levical Socreties.}

\section{ROYAL MEDICAL AND CHIRURGICAL SOCIETY.} TUESDAT, NOVEMBER 14TH, 1871.

Dr. H. A. Pitman, Viog-President, in the Chatr.

ON THE OPERATION OF OPENING THE LARYNX BY SECTION OF THE CARTILAGES, \&o, IN ORDER TO FACILITATE THE REMOVAL OF MORBID GROWTHS.

BY ARTHUR E. DURHAM, F.R.C.S.

ASSYSTANT-SURGION TO, AND LECTURER ON ANATOMY AT, GUY'S HOSPITAL.

The anthor related in detail five cases in each of which this operation had been performed in Guy's Hospital: in three cases by himself, in one by Mr. Bryant, and in one by Mr. Colley. The results in four of these cases had been eminently satisfactory, free respiration and good voice having been regained. The remaining case was still under treatment.

Appended to this communication were more or less complete reports of all the cases the author had been able to find on record. These cases were 32 in number, and with the 5 detailed in his communication gave a total of 37 . In 19 of these the operation might be regarded as having been completely successful, natural respiration and voice (though in some instances not normal in tone) having been restored. In 7 partial success was obtained, respiration having been restored, but the voice lost or very seriously impaired. In 4 cases some temporary relief was obtained. In 3 the result might be considered negative, neither good nor harm having been done. The reports of at least 2 were incomplete. In 2 cases-and two cases only-death resulted. In each of these, however, the immediate cause was blood-poisoning. Metastatic abscesses were found in the lungs in the one case; in the other erysipelas and gangrene occurred, and broncho-pneumonia and exhaustive fever ensued, and led to the fatal issue.

Comparing the results thus stated with those given by Dr. Mackenzie in his Treatise on Growths in the Larynx, the author pointed out that death could properly be attributed to the operation in two only out of the nine cases enumerated by Mackenzie as having terminated fatally. these two being the same as those already alluded to. With regard to the other seven cases the author specified each, and showed that in each the result of the operation 